\title{
Human Exploration of Mars Design Reference Architecture 5.0
}

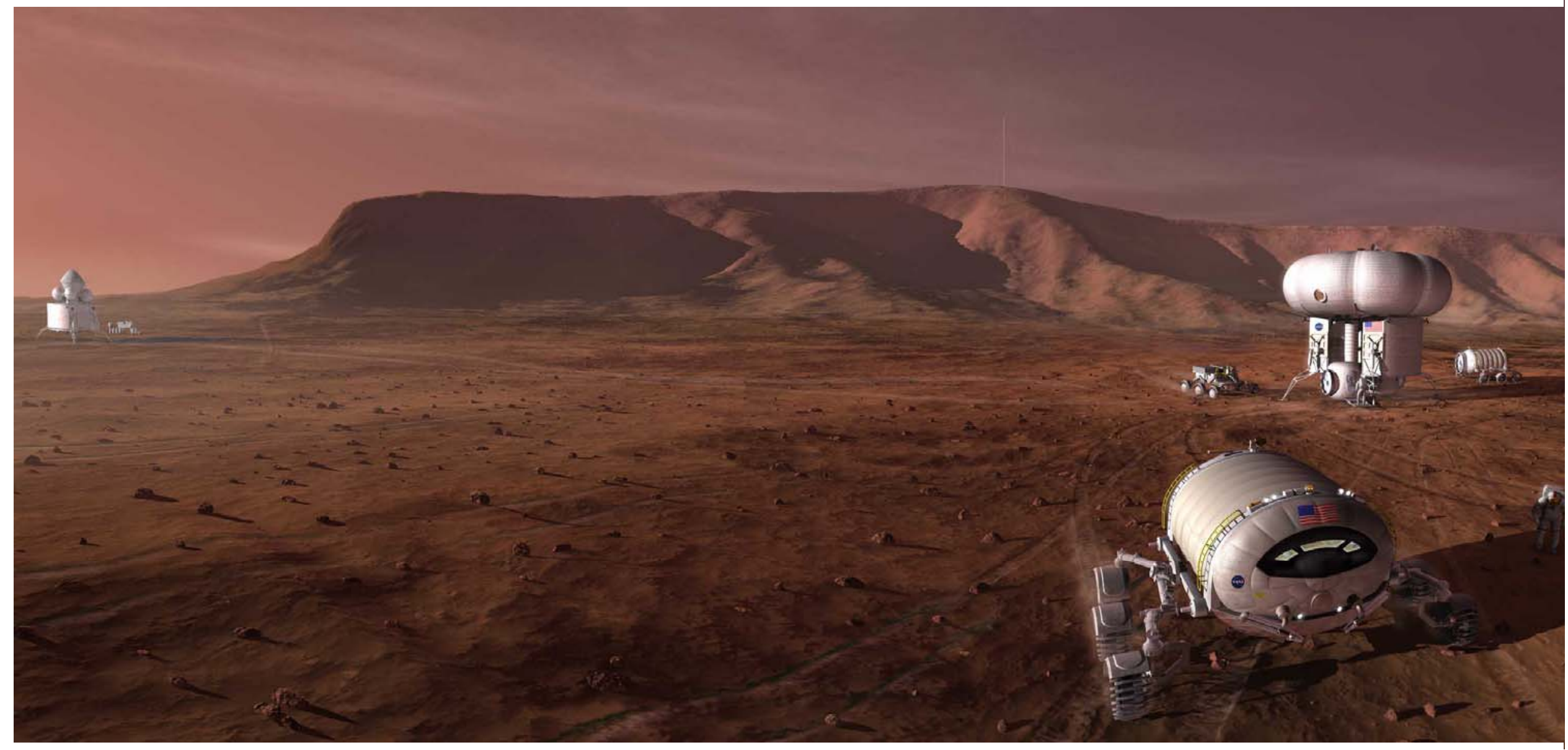

Bret G. Drake

Lyndon B. Johnson Space Center

February 2009 


\section{Mars Design Reference Mission Evolution and Purpose}

- Exploration mission planners maintain "Reference Mission" or "Reference Architecture"

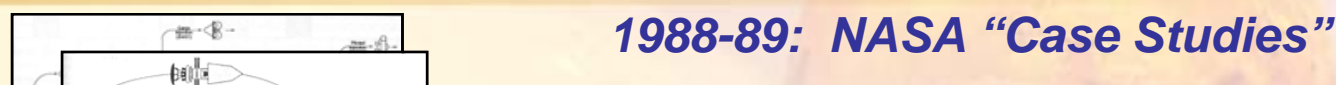

- Represents current "best" strategy for human missions

- The Mars DRA is not a formal plan, but provides a vision and context to tie current systems and technology developments to potential future missions

- Also serves as benchmark against which alternative architectures can be measured

- Constantly updated as we learn

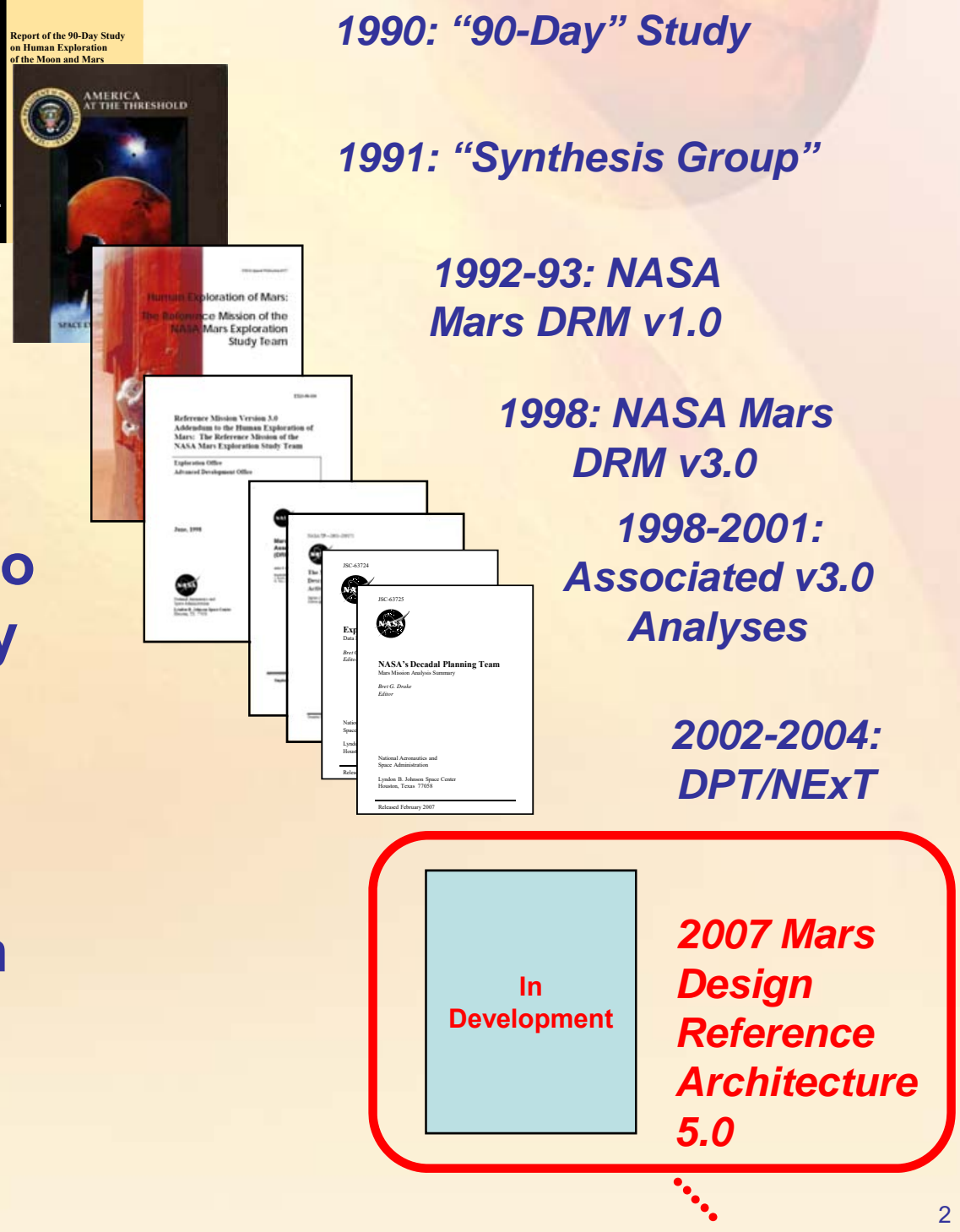




\section{Study Objectives / Products}

- Update NASA's human Mars mission reference architecture, that defines:

- Long term goals and objectives for human exploration missions

- Flight and surface systems for human missions and supporting infrastructure

- Current Constellation systems and other systems updated since Mars DRM 4.0 (circa 1998)

- Update and incorporate Mars surface reference mission into current strategy

- An operational concept for human and robotic exploration of Mars

- Key challenges including risk and cost drivers

- Development schedule options (deferred)

- Assess strategic linkages between lunar and Mars strategies

- Develop an understanding of methods for reducing the cost/risk of human Mars missions through investment in research, technology development and synergy with other exploration plans, including:

- Robotic Mars missions, Cis-lunar activities, ISS activities, Earth-based activity, including analog sites, laboratory studies, and computer simulations, additional research and technology development investment

- Develop a forward plan to resolve issues not resolved during 2007 


\section{Mars Design Reference Architecture 5.0 Study Approach}

- Non-Science Requirements

- Systems Development

- Human Exploration Architecture

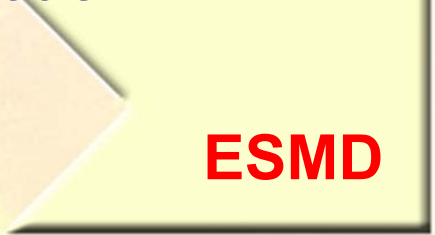

- Science Requirements

- Integration with ongoing Mars Exploration Program

- Interpretation of science SMD results

\section{Mars Design Reference Architecture 5.0}

Science Community (Mars Exploration

Program Analysis Group

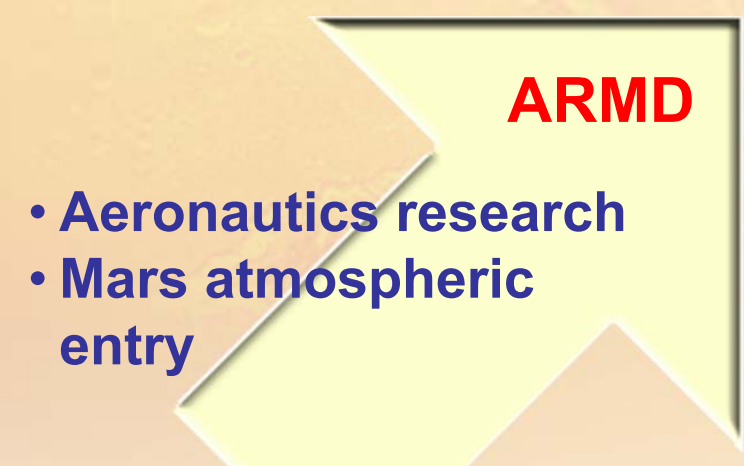

SOMD

- Human Spaceflight Operations

- Tracking, navigation and communications

- Integrating all stakeholders while leveraging recognized subject matter experts

- Mission Directorates will assign and provide funding for personnel within their respective directorates 


\section{Study Organizational Structure}

\section{Joint Steering Group}

- Agency Guidance and Decision Concurrence

- Exploration Systems Mission Directorate

- Science Mission Directorate

- Aeronautics Research Mission Directorate

- Space Operations Mission Directorate

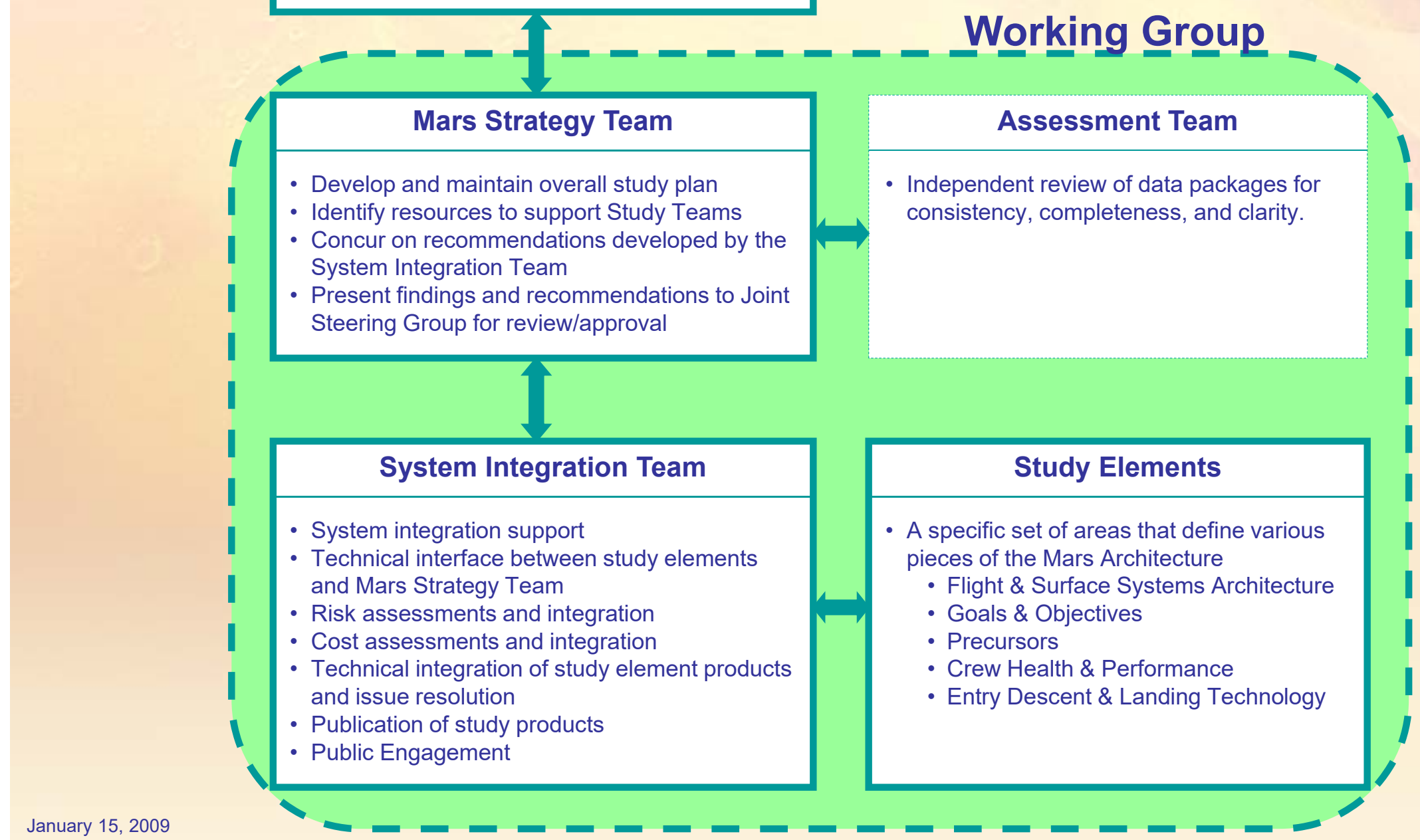

\section{Mars Architecture} Working Group 


\section{Trade Tree Trimming Objectives}

- Reduce the number of trade options

- Utilize step-wise decision approach similar to that used by the Exploration Systems Architecture Study (ESAS)

- Concentrate on those key trade tree branches which provide the most architectural leverage. Equal emphasis on cost, risk, and performance

- Ensure proper fidelity is matched with decision confidence. That is, concentrate on those items which "make a difference".

- Continue to refine the approach as decisions are made and increased fidelity is achieved 


\section{Mars Design Reference Architecture 5.0 Refinement Process}

- Phase I: Top-down, High-level - Mission Design Emphasis

- Focus on key architectural drivers and key decisions

- Utilization of previous and current element designs, ops concepts, mission flow diagrams, and ESAS risk maturity approach information where applicable

- Narrow architectural options (trimming the trade tree) based on risk, cost and performance

- First order assessments to focus trade space on most promising options for Phase II

- Phase II: Strategic With Emphasis on the Surface Strategy

- Refinement of leading architectural approach based on trimmed trade tree

- Elimination of options which are proven to be too risky, costly, or do not meet performance goals

- Special studies to focus on key aspects of leading options to improve fundamental approach

- Propose basic architecture decisions 


\section{Mars Design Reference Architecture 5.0 2007 Key Decision Packages}

\section{Mission Type:}

Which mission type, conjunction class (long surface stay) or opposition class (short surface stay) provides the best balance of cost, risk, and performance?

\section{Pre-Deployment of Mission Cargo:}

Should mission assets, which are not used by the crew until arrival at Mars, be pre-deployed ahead of the crew?

3. Mars Orbit Capture Method

Should the atmosphere of Mars be used to capture mission assets into orbit (aerocapture)?

4. Use of In-Situ Resources for Mars Ascent

Should locally produced propellants be used for Mars ascent?

5. Mars Surface Power Strategy

Which surface power strategy provides the best balance of cost, risk, and performance? 


\section{Mars Design Reference Architecture 5.0 Top-level Trade Tree}

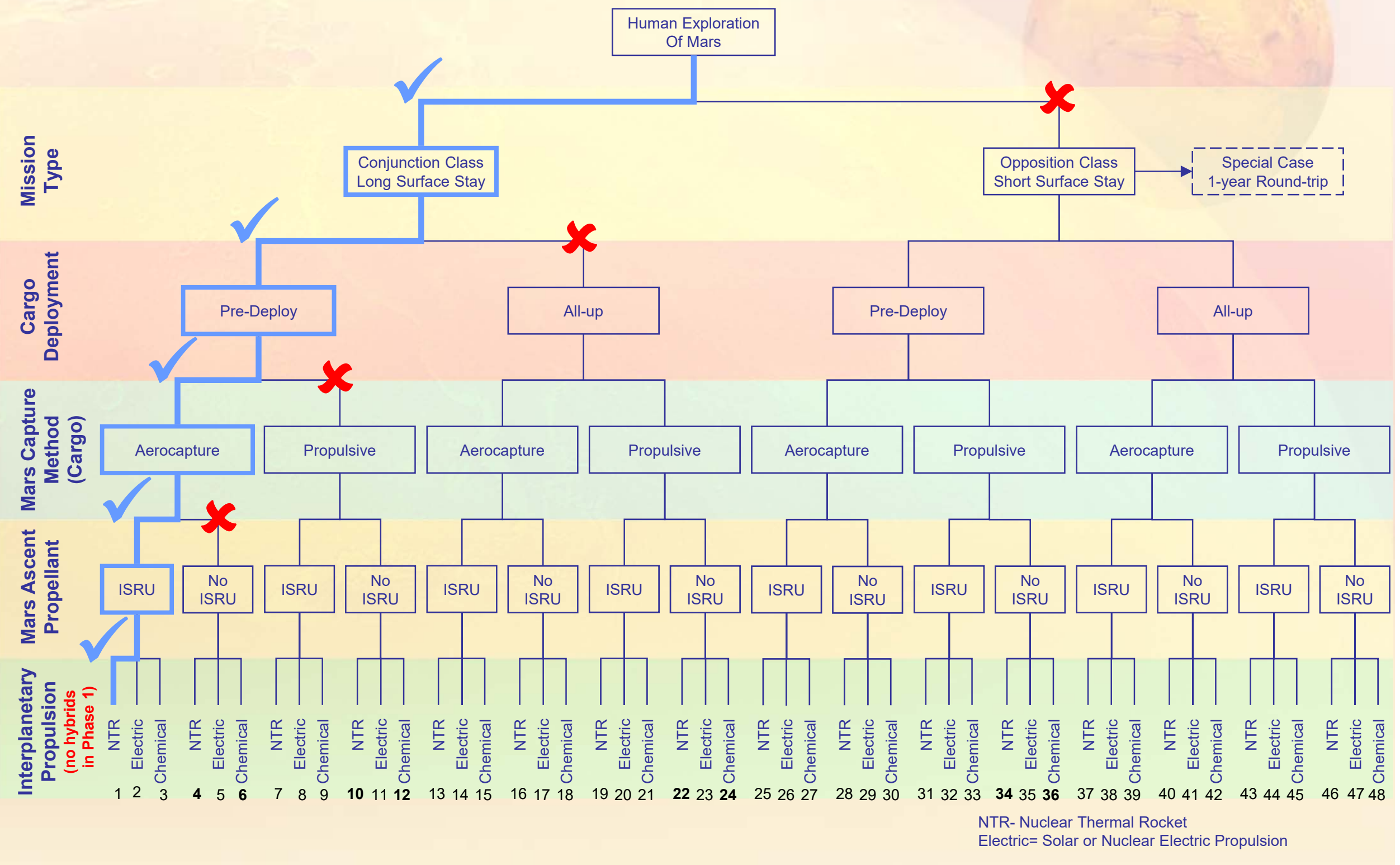




\section{How to Capitalize on the Unique Attributes of Human Explorers}

- Human explorers bring unique abilities to exploration:

- Cognition

- Rapidly recognize and respond to unexpected findings; sophisticated, rapid pattern recognition (structural/morphological biosignatures).

- Dexterity

- Humans are capable of lifting rocks, hammering outcrops, selecting samples, etc..

- Adaptability

- Humans are able to react in real time to new and unexpected situations, problems, hazards and risks.

- Efficiency

- Sample and equipment manipulation and problem solving.

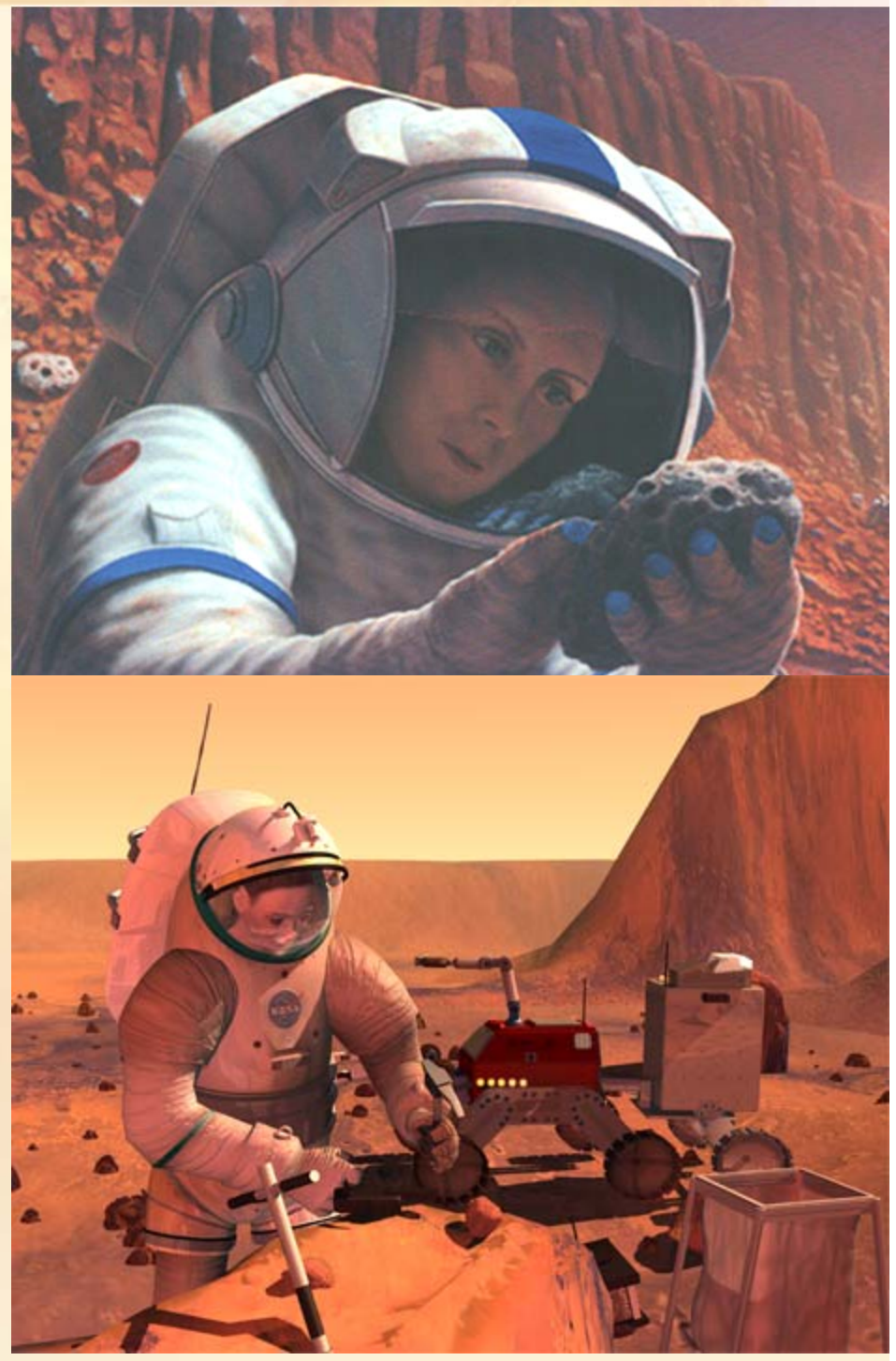




\section{Possible Objectives Program of First Three Human Missions}

- Goals for initial human exploration of Mars organized into the following taxonomy:

\begin{tabular}{|c|l|}
\hline Goal I & $\begin{array}{l}\text { Potential for Life (MEPAG - Mars } \\
\text { Exploration Program Analysis Group) }\end{array}$ \\
\hline Goal II & Current and ancient climate (MEPAG) \\
\hline Goal III & Geology \& geophysics (MEPAG) \\
\hline
\end{tabular}

\begin{tabular}{|c|l|}
\hline Goal IV & Preparation for human exploration (MEPAG - update pending \\
\hline Goal IV+ & Preparation for sustained human presence (ESMD) \\
\hline Goal V & Ancillary science (SMD) \\
\hline
\end{tabular}

- Relationship between the resulting goals and proposed implementation approaches addressed:

- Different exploration sites or same site?

- Short stay (30-day) or long stay (500-days)

- Recommendation:

- Long-stay missions overwhelmingly preferred

- Multiple sites preferred from a science perspective

- Same site probably better for sustained presence

- Maximize mobility, on-Mars field (and field lab) science capability, and options for returned sample science

Two different sets of priorities for key program attributes from different stakeholders

PLANETARY SCIENCE

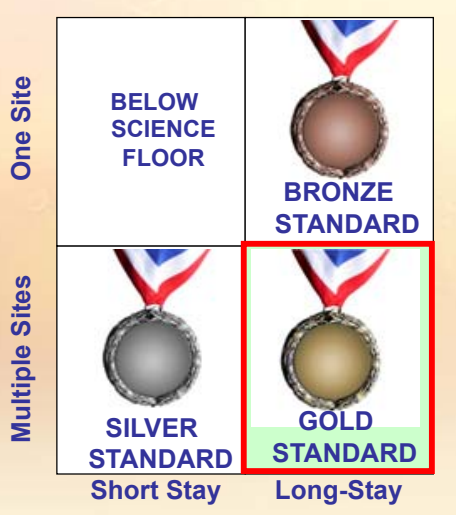

SUSTAINED PRESENCE

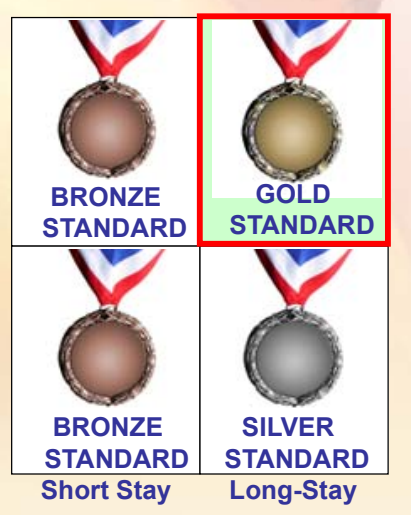




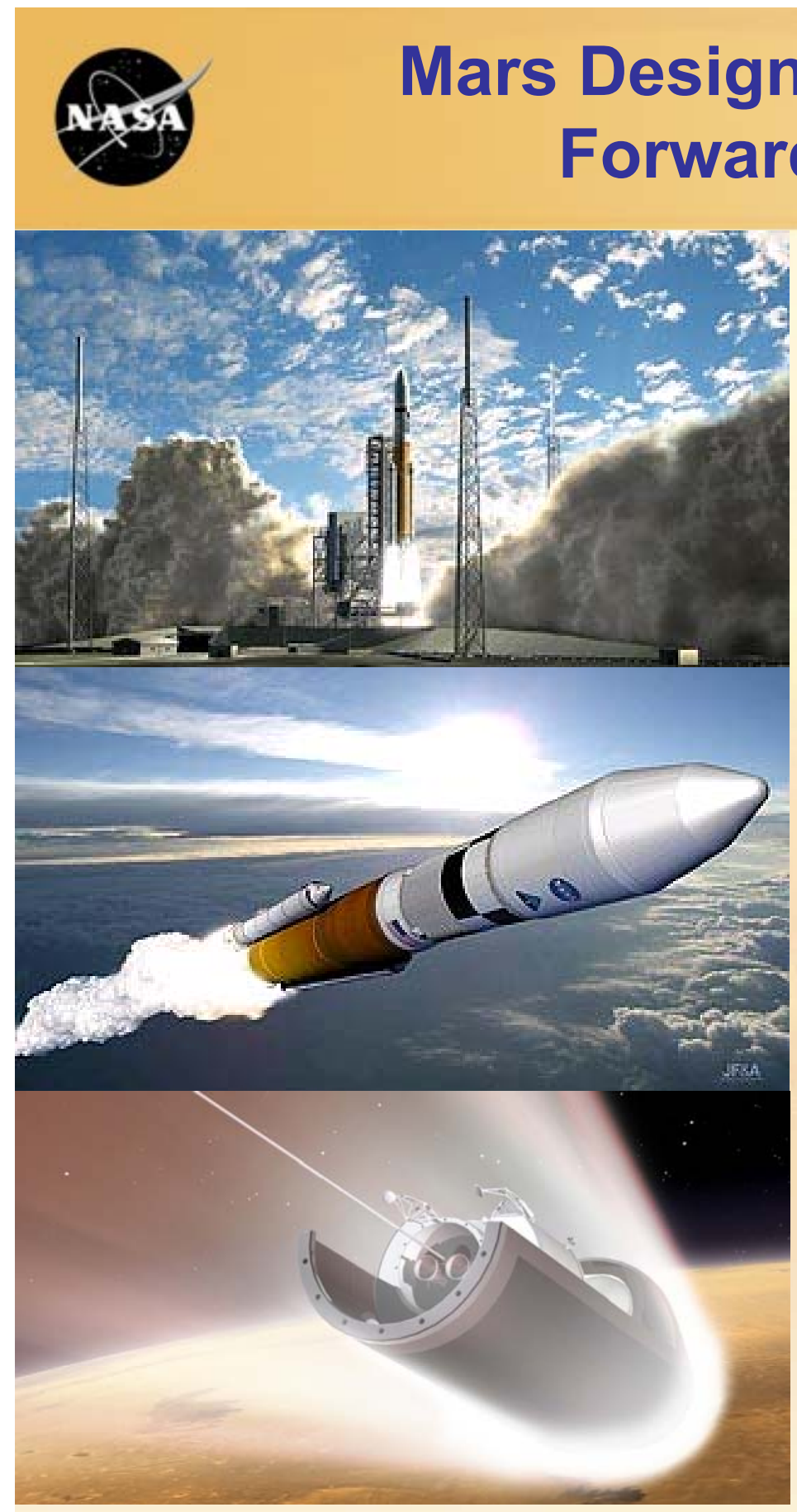

- Twenty-six months prior to crew departure from Earth, pre-deploy:

- Mars surface habitat lander to Mars orbit

- Mars ascent vehicle and exploration gear to Martian surface

- Deployment of initial surface exploration assets

- Production of ascent propellant (oxygen) prior to crew departure from Earth

- Six crew travel to Mars on "fast" (six month) trajectory

- Reduces risks associated with zero-g, radiation

- Rendezvous with surface habitat lander in Mars orbit

- Crew lands in surface habitat which becomes part of Mars infrastructure

- Sufficient habitation and exploration resources for 18 month stay 


\section{Mars Design Reference Architecture 5.0 Mission Profile NTR Reference Shown}

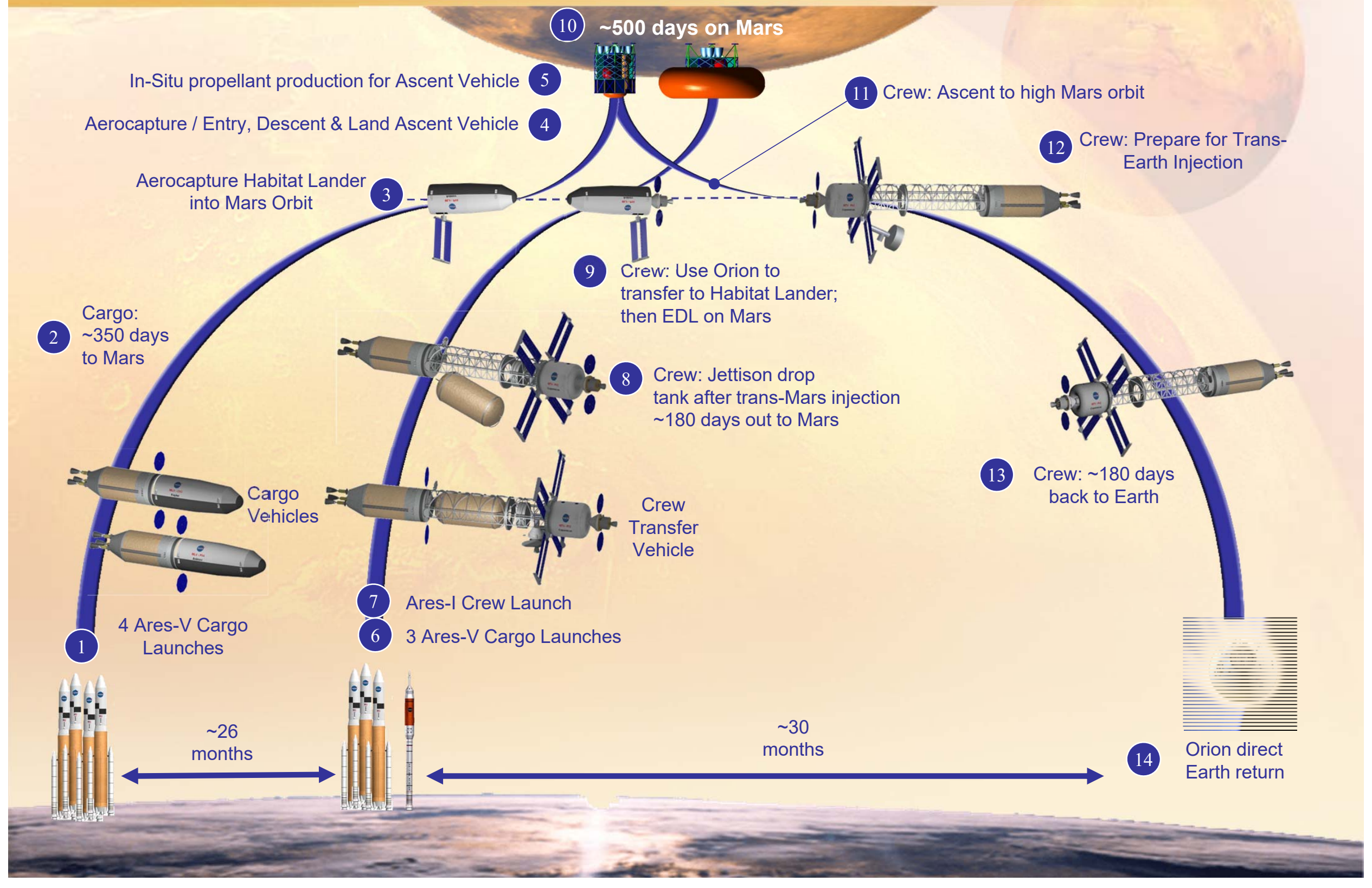




\section{Mars Design Reference Architecture 5.0 Flight Sequence}

\section{- Long-surface Stay + Forward Deployment}

- Mars mission elements pre-deployed to Mars prior to crew departure from Earth

- Surface habitat and surface exploration gear

- Mars ascent vehicle

- Conjunction class missions (long-stay) with fast inter-planetary transits

- Successive missions provide functional overlap of mission assets
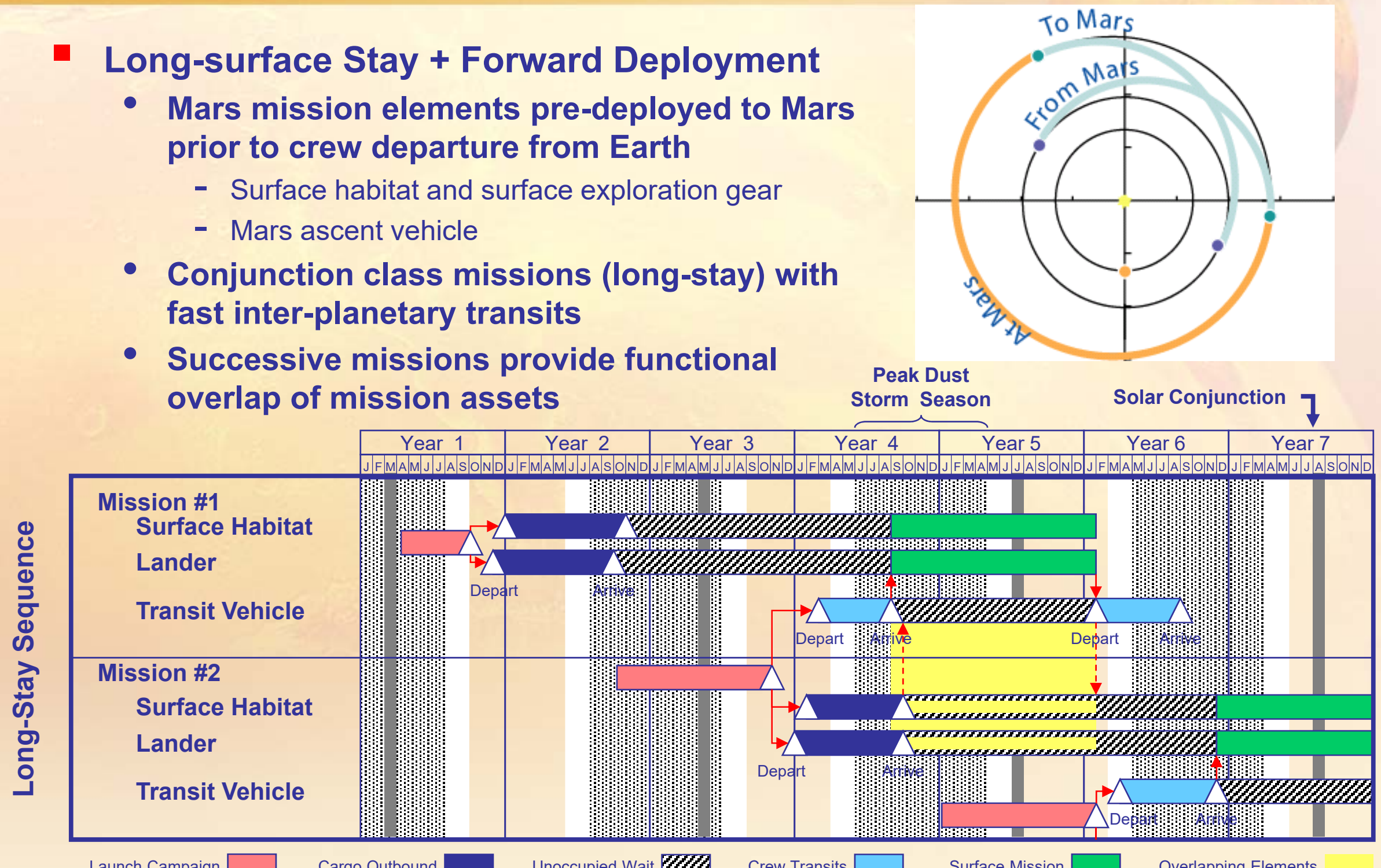

Launch Campaign

Cargo Outbound

Unoccupied Wait $\mathscr{W}$

Crew Transits

Surface Mission

Overlapping Elements 


\section{Human Exploration A Historical Perspective}

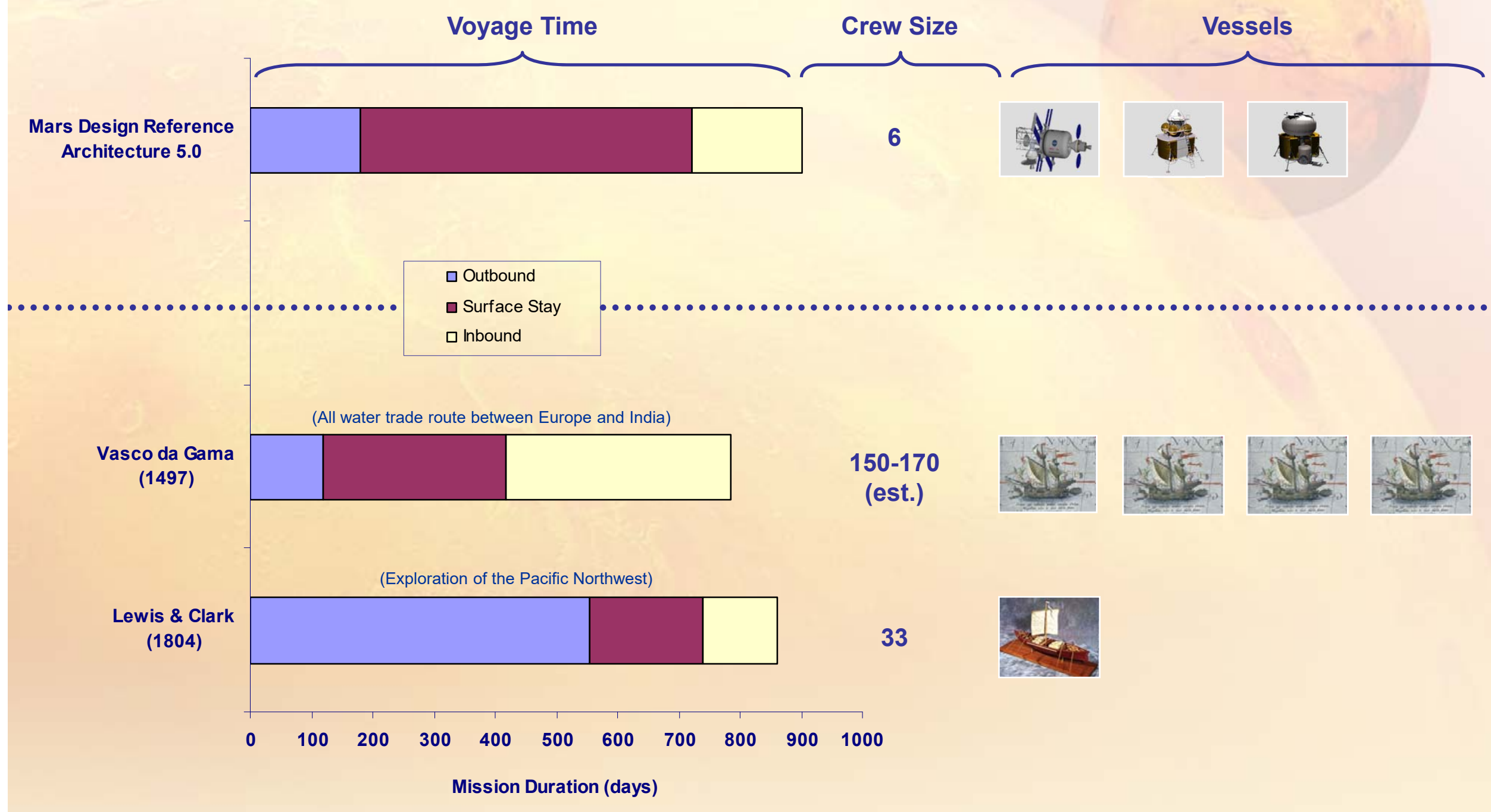

Human mission to Mars will be long and complex, but the round trip duration is within the experience of some of the most successful exploration missions with significantly far fewer crew 


\section{Ares V Elements \\ - Recommended POD (51.0.48) -}

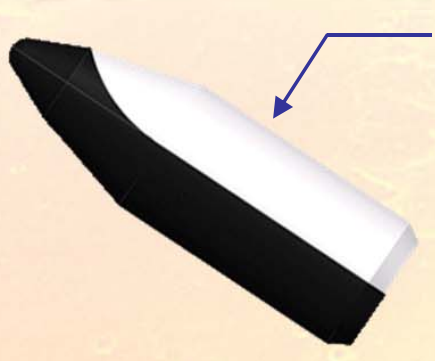

Shroud (Dual use shroud shown)

- 10 m (dia) x 30 m (length)

- Dual use shroud used as entry system at Mars

- Standard or nosecone only versions for other Mars payload elements

Mars Payload
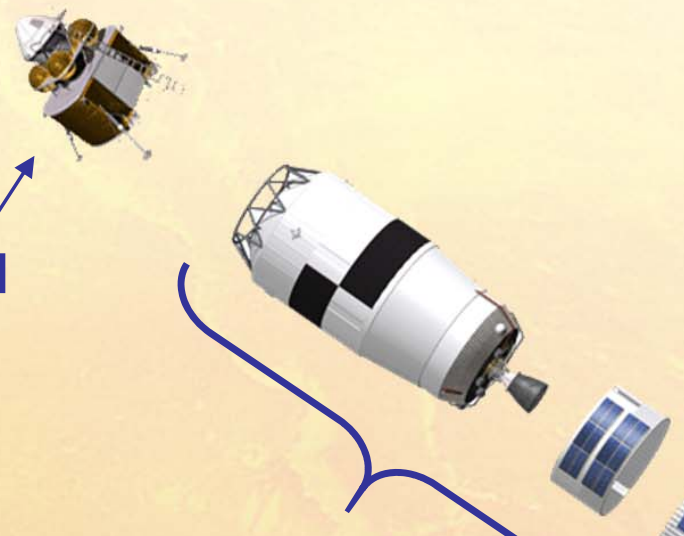

\section{Performance to LEO}

- Jettison shroud Option:

Payload

$130.8 \mathrm{t}$

- Dual Use shroud:

Payload:

$83.6 \mathrm{t}$

Shroud:

$50.0 \mathrm{t}$

Solid Rocket Boosters (2)

- Two recoverable 5.5-segment PBAN-fueled, steel-casing boosters (derived from current Ares I first stage

Earth Departure Stage (EDS)

- Loiter skirt eliminated (no loiter required for Ares $\mathrm{V}$ )

- $\sim 40 \%$ Propellant offloaded

Core Stage

- Six Delta IV-derived RS-68 LOX/LH $\mathrm{L}_{2}$ engines (expendable)

- 10 m (33 ft) diameter stage

- Composite structures

- Aluminum-Lithium (Al-Li) tanks

RS-68B Engines

(6) 


\section{Cargo Vehicle Descent / Ascent Vehicle (DAV)}

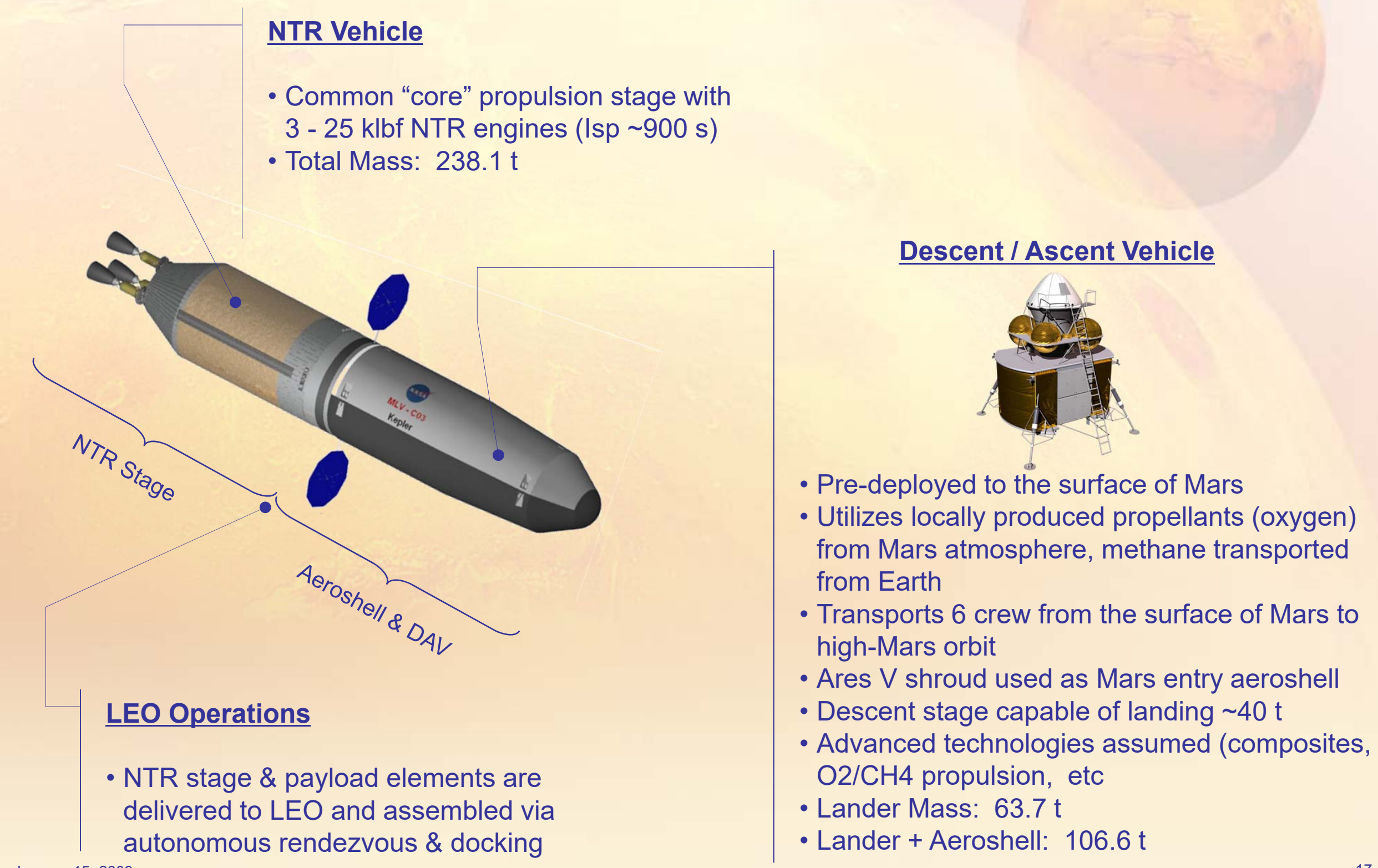




\section{Cargo Vehicle Surface Habitat (SHAB)}

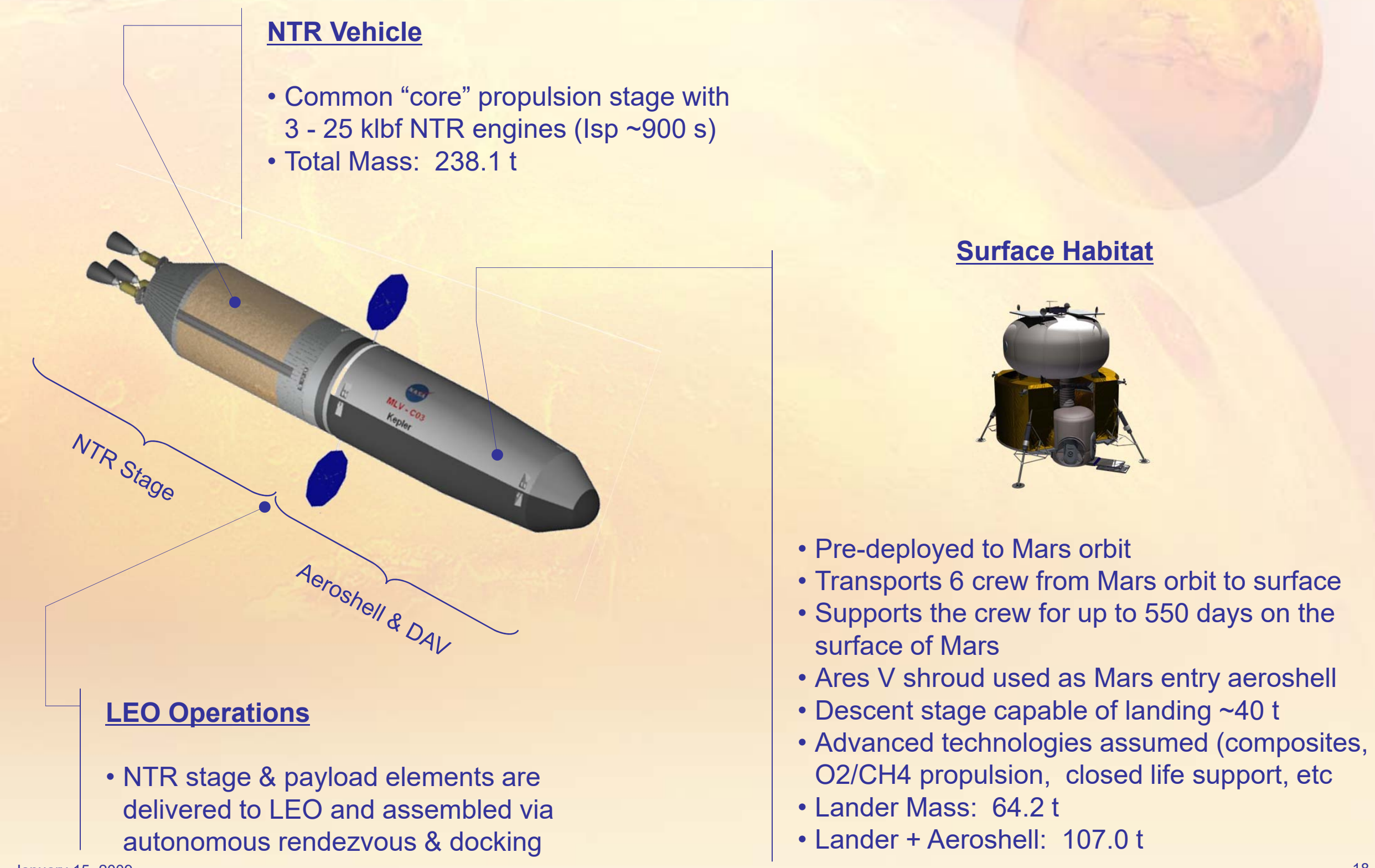




\section{Crew Vehicle Mars Transit Vehicle (MTV)}

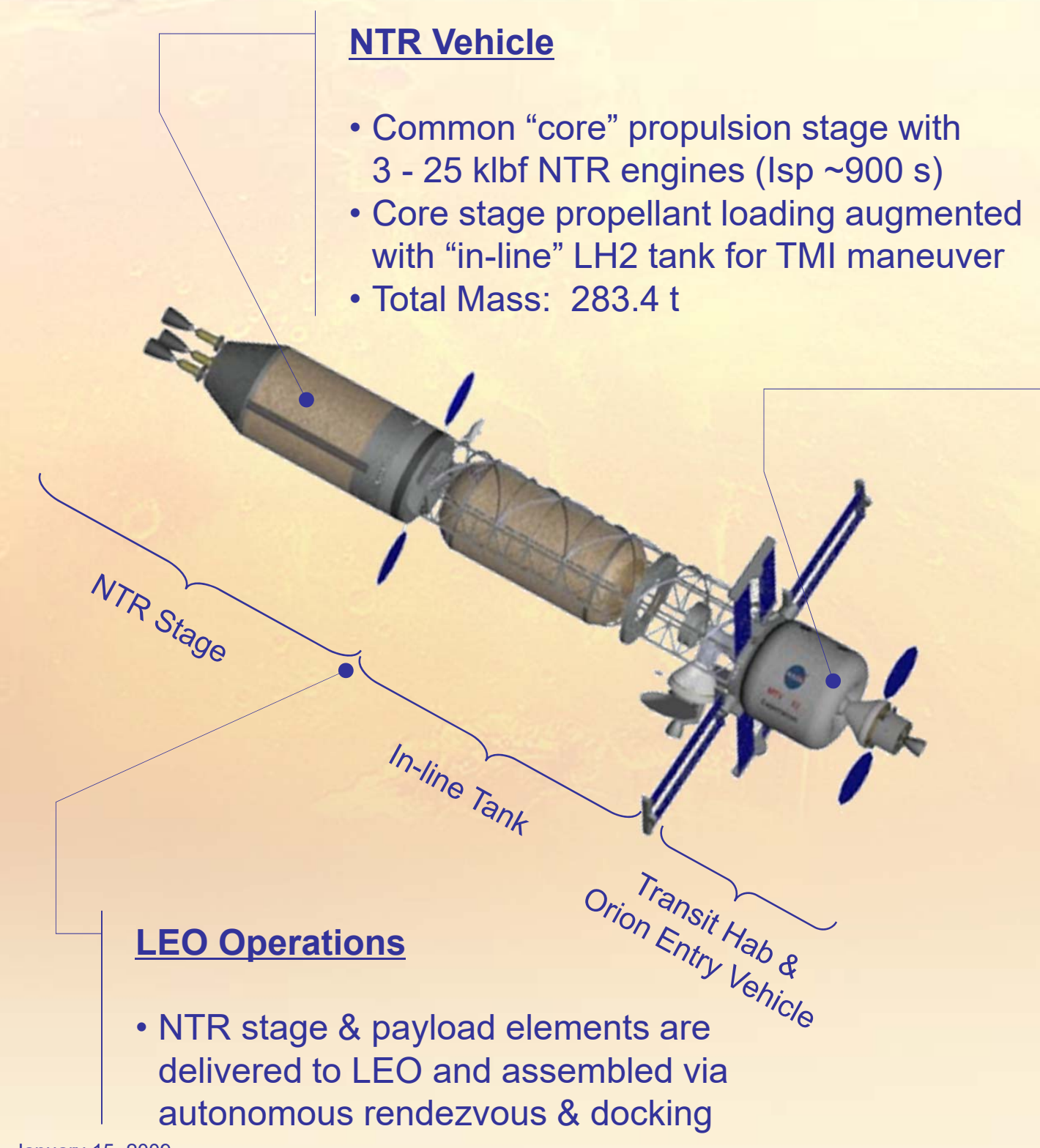

\section{Transit Habitat \& Orion Entry Vehicle}

- Transports 6 crew round trip from LEO to high-Mars orbit and return

- Supports 6 crew for 400 days (plus 550 contingency days in Mars orbit)

- Crew direct entry in Orion at $12 \mathrm{~km} / \mathrm{s}$

- Advanced technologies assumed (composites, inflatables, closed life support, etc

- Transit Habitat Mass: $41.3 \mathrm{t}$

- Orion: $10.0 \mathrm{t}$ 


\section{Orion Crew Transfer / Earth Return Vehicle}

- Crew Delivery to LEO (Block 1)

- Provide safe delivery of 6 crew to Earth orbit (for rendezvous with the MTV)

- Delivery and return of checkout crew prior to the mission

- Delivery of the mission crew

- ISS Block1 configuration

- End of Mission Crew Return (Mars Block)

- Provide safe return of $6 \mathrm{crew}$ from the MarsEarth transfer trajectory to Earth at the end of the mission

- $12 \mathrm{~km} / \mathrm{s}$ entry speed

- 900 day dormant operations

- 3 day active operations

- Much smaller service module ( $\sim 300 \mathrm{~m} / \mathrm{s}$ delta- $\mathrm{v})$ for re-targeting and Earth entry corridor set-up
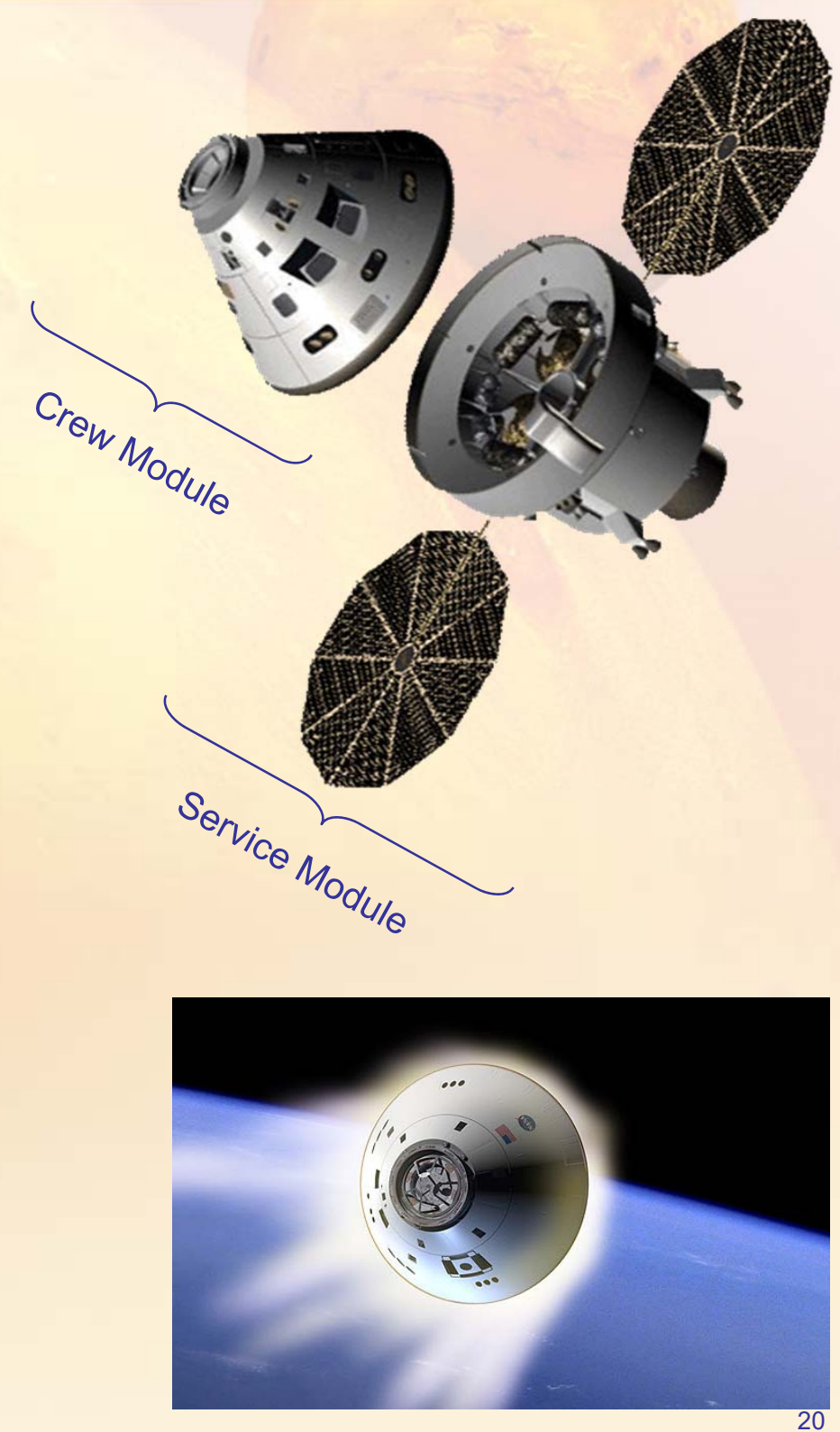


\section{Mars Design Reference Architecture 5.0 Surface Strategy Options}

- Multiple strategies developed stressing differing mixes of duration in the field, exploration range, and depth of sampling

- Mobile Home: Emphasis on large pressurized rovers to maximize mobility range

DRA 5.0 Reference

- Commuter: Balance of habitation and small pressurized rover for mobility and science

- Telecommuter: Emphasis on robotic exploration enabled by teleoperation from a local habitat

- Mobility including exploration at great distances from landing site, as well as sub-surface access, are key to Science Community

- In-Situ Consumable Production of life support and EVA consumables coupled with nuclear surface power provides greatest exploration leverage

- Development of systems which have high reliability with minimal human interaction is key to mission success

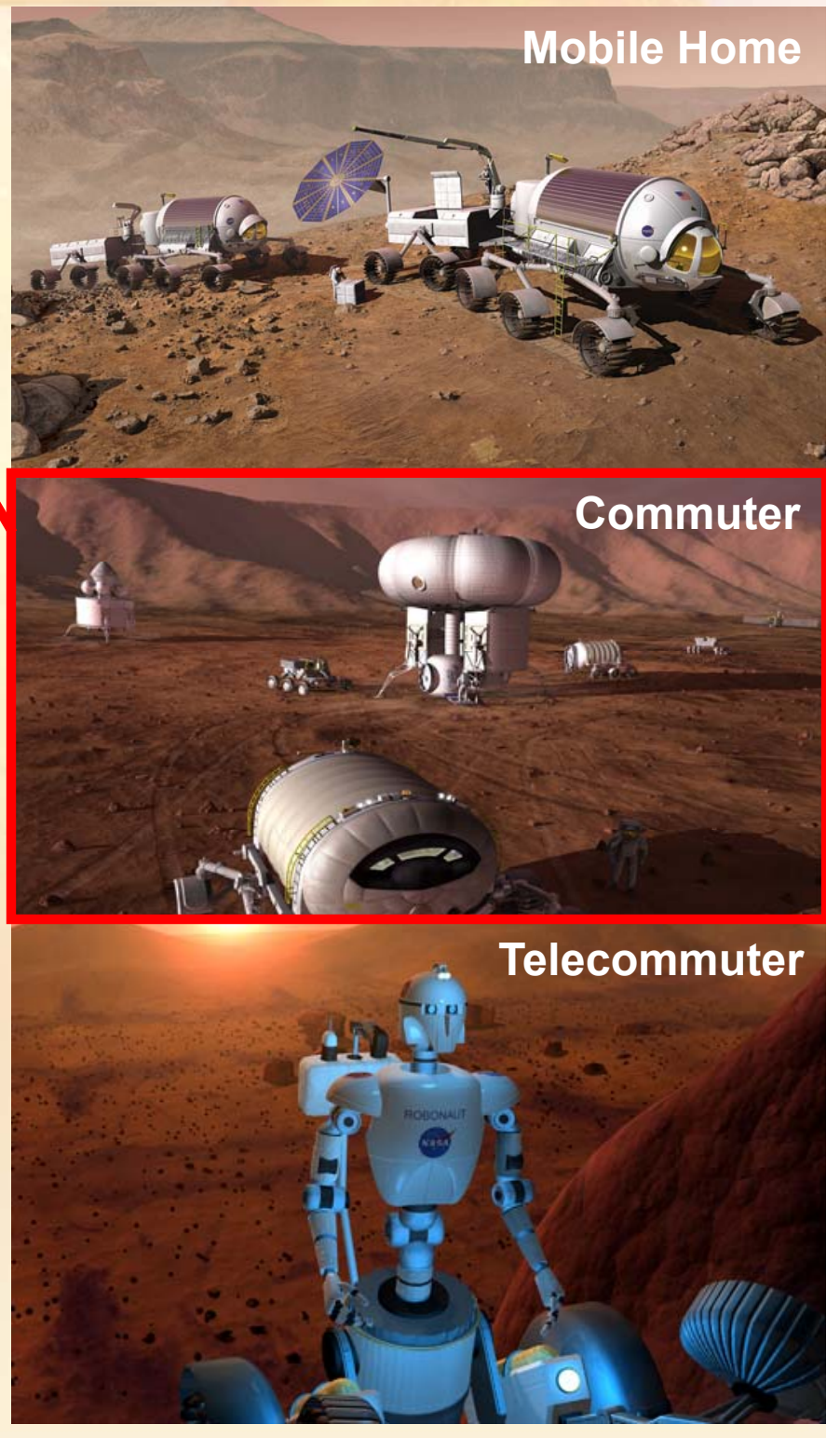




\section{Mars Design Reference Architecture 5.0 Ground Operations}

- Maintaining a launch frequency greater than $\mathbf{3 0}$ days eliminates the need for a new Ares V Pad.

- Mars launch campaign requires a new Offline SRB Stacking Facility*, two additional Ares V Mobile Launchers and one additional Ares V Integration Cell in the $\mathrm{VAB}^{* *}$.

- Providing adequate launch schedule margin (additional 3-6 months prior to TMI window opening) is key to maximizing mission success.

- NTR likely will require a new Processing Facility.

- Chemical option assumes a limited Ground Processing concept (TMI, MOI and TEI modules) similar to current CLV Upper stage and Ares V EDS concepts.

- Surface Nuclear Power will likely require a new processing facility (if NTR, use same facility planned for processing the Common Core Stage).

* A new 'VAB like' Ares Vertical Integration Facility and adding additional Ares V Mobile Launchers were also studied.

** New Ground System assets above what is currently planned for Lunar Missions

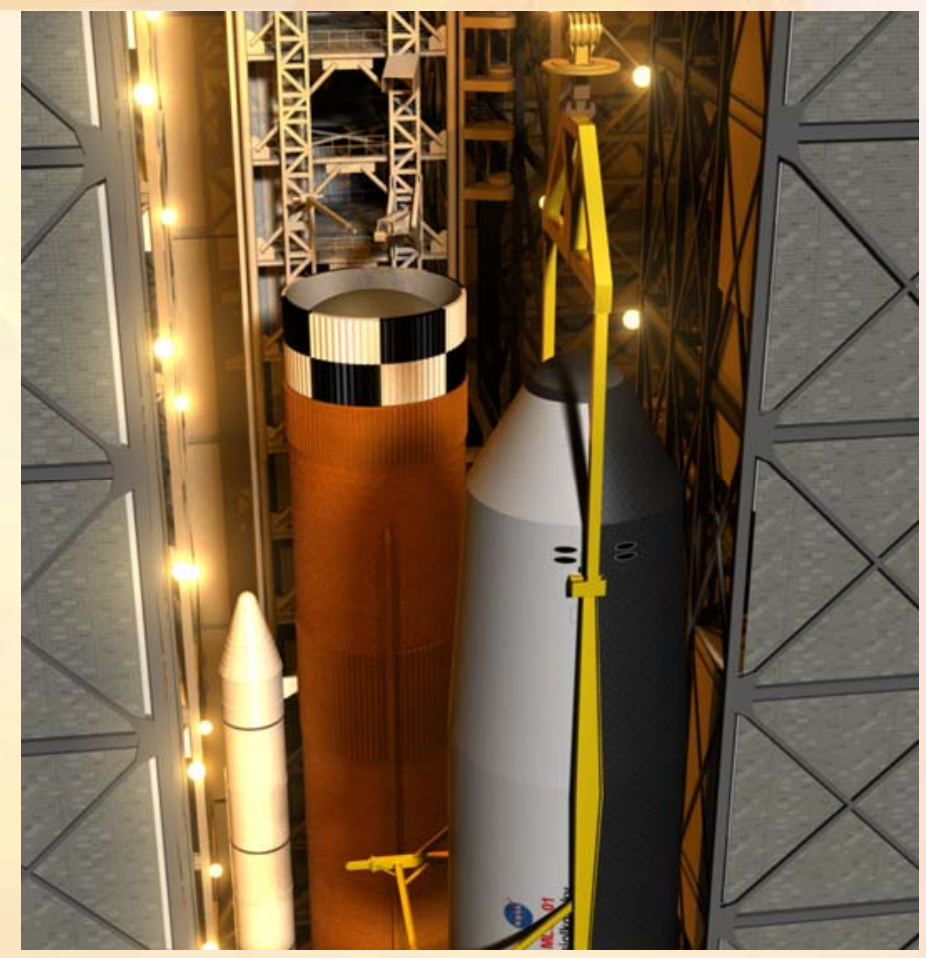




\section{Mars Design Reference Architecture 5.0 Entry, Descent \& Landing (EDL)}

- Aerocapture of uncrewed cargo vehicles continues to remain the leading option since they already have aeroshells

- Recommend retaining propulsive capture of crew elements (technical mass and packaging issues)

- First use of EDL identified as a key risk driver (scalable/near-full scale precursor will help retire risk)

- Landing of large payloads (greater than $2 t$ ) on the surface of Mars remains a key challenge (supersonic transition problem)

- Research and system studies of fundamental EDL is highly recommended

- Thorough EDL risk mitigation strategy, including robotic mission demonstration and use of EDL systems which are scalable/near-full scale to human mission needs is highly recommended

- Aborts during EDL flight phase highly unlikely. Further assessments required, but continue to stress Abort to Surface strategies.

- Limiting Earth return to less than $12 \mathrm{~km} / \mathrm{s}$ will keep TPS near "within Orion Family".

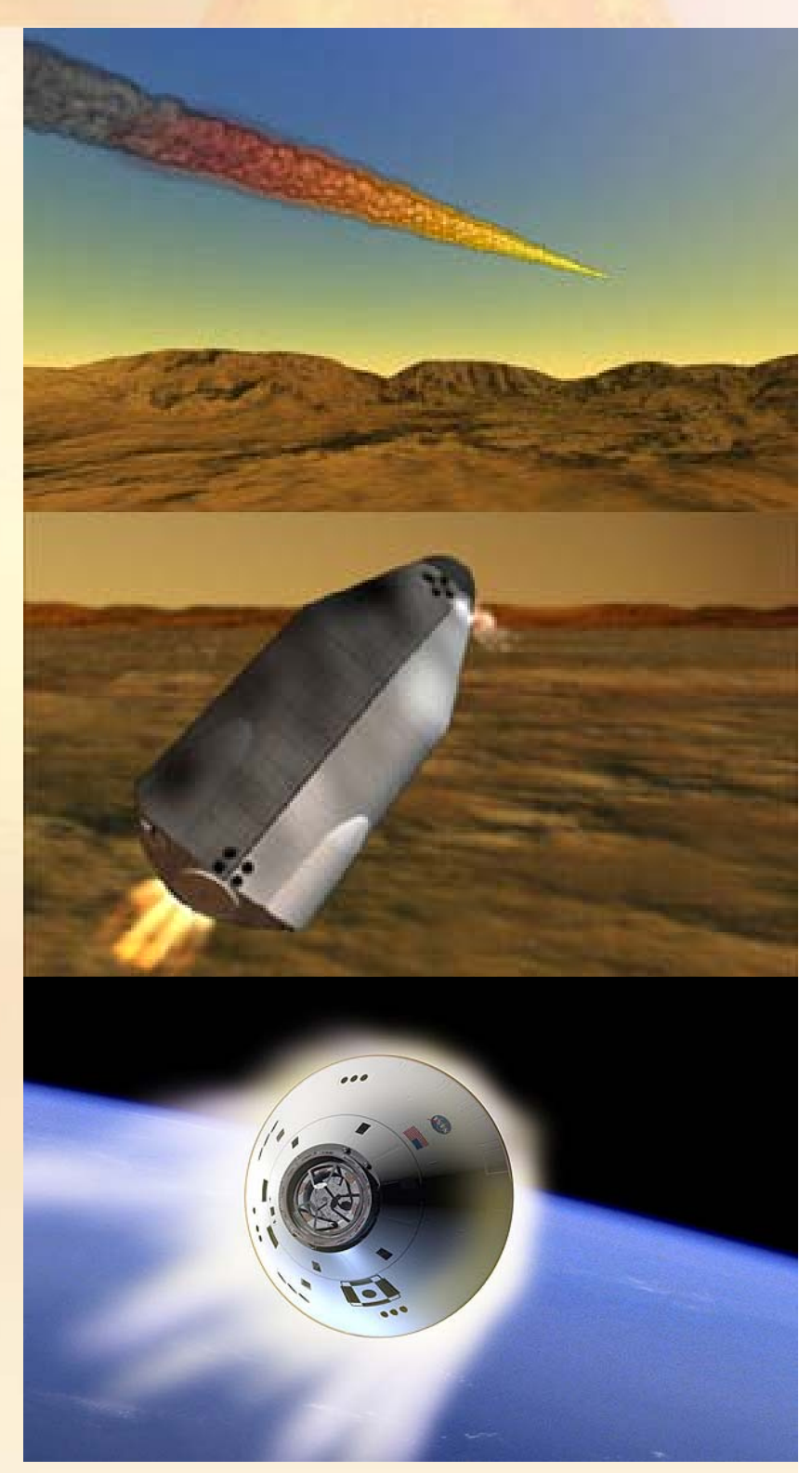




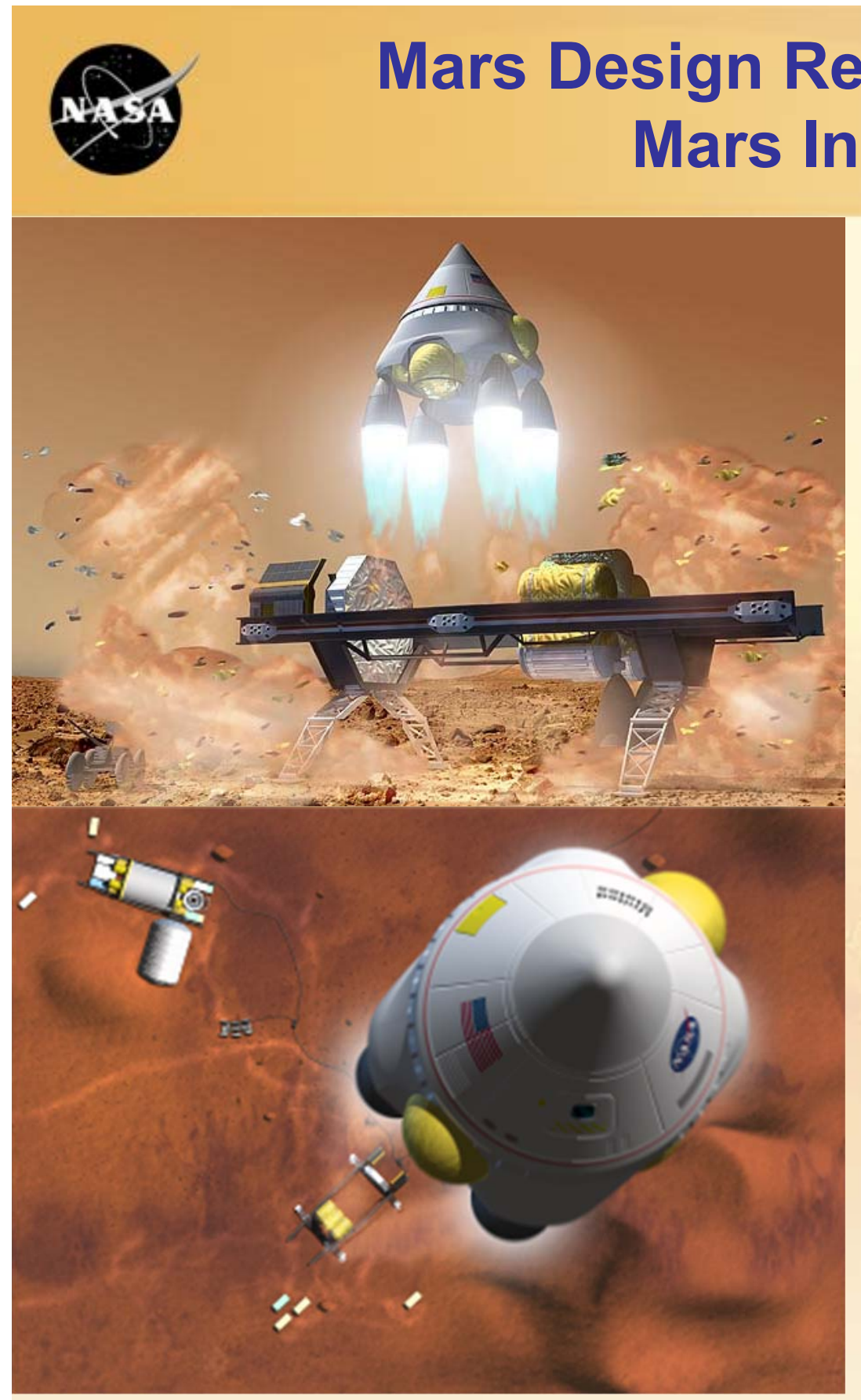

- ISRU commodities produced prior to crew departure from Earth

- Commodity handling is a subset of proven lunar ISRU techniques

- Propellants (oxygen) used for ascent from surface to High-Mars Orbit produced from Mars atmosphere

- $30 \%$ reduction in lander mass

- Reduction in volume thus easing packaging of lander in the aeroshell

- Producing caches of water and oxygen provides backup to life support systems

- Can reduce level of closure (and expense) of surface life support systems

- Technical risk can be mitigated by lunar and robotic mission tests of Martian resource extraction

- Could also make sense as a sample return strategy 


\section{Design Reference Architecture 5.0 Surface Power System}

- $30 \mathrm{kWe}$ fission power system supports ISRU (prior to crew arrival) and during crew exploration

- Reactor deployed $1 \mathrm{~km}$ from lander remotely

- Close derivative of the lunar system
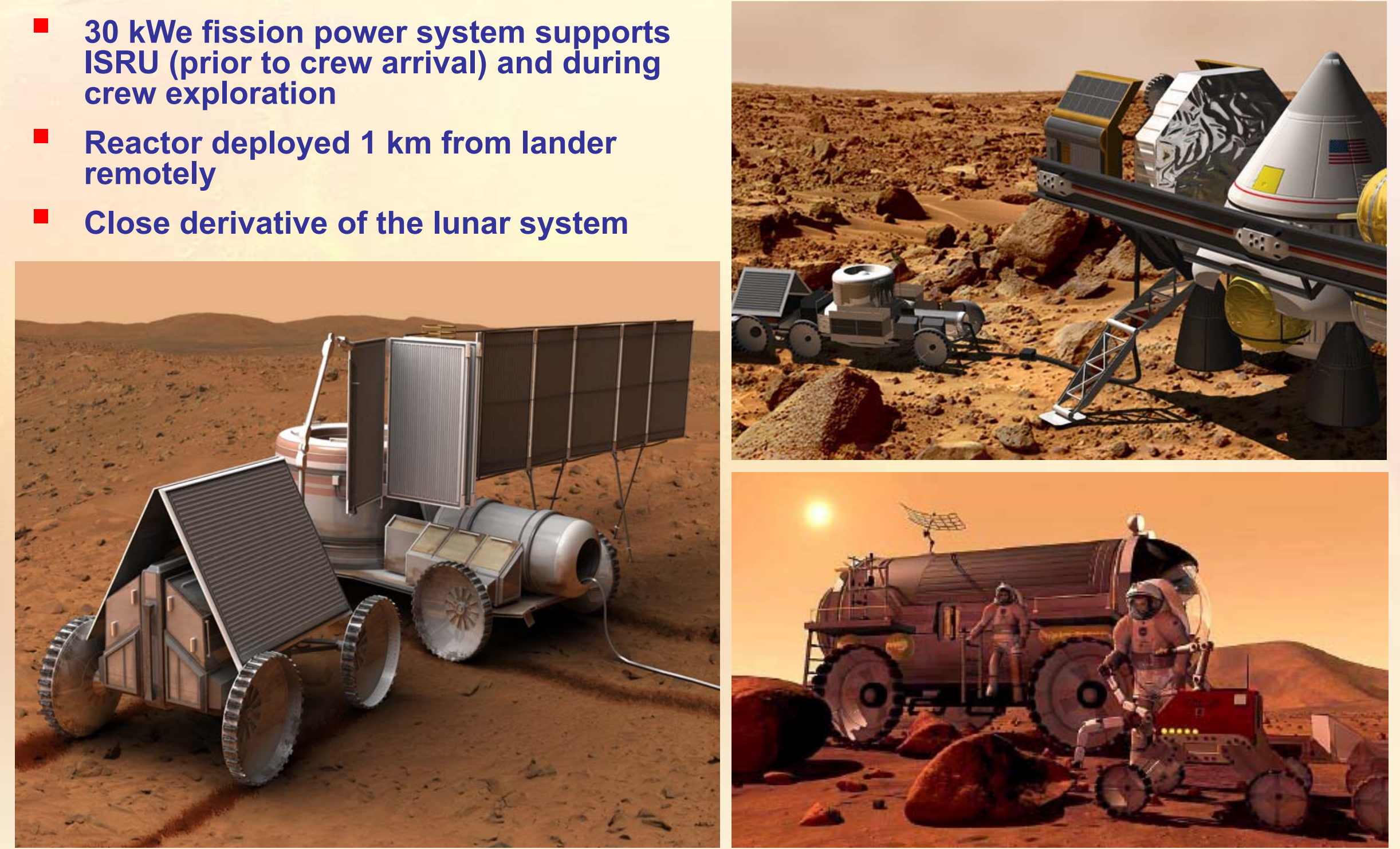


\section{Mars Design Reference Architecture 5.0 Surface Exploration and Discovery}

- Long surface stays with visits to multiple sites provides scientific diversity thus maximizing science return

- Sustainability objectives favor return missions to a single site (objectives lend themselves best to repeated visits to a specific site on Mars)

- Mobility at great distances (100's km) from the landing site enhances science return (diversity)

- Subsurface access of 100's m or more highly desired

- Advanced laboratory and sample assessment capabilities necessary for high-grading samples for return

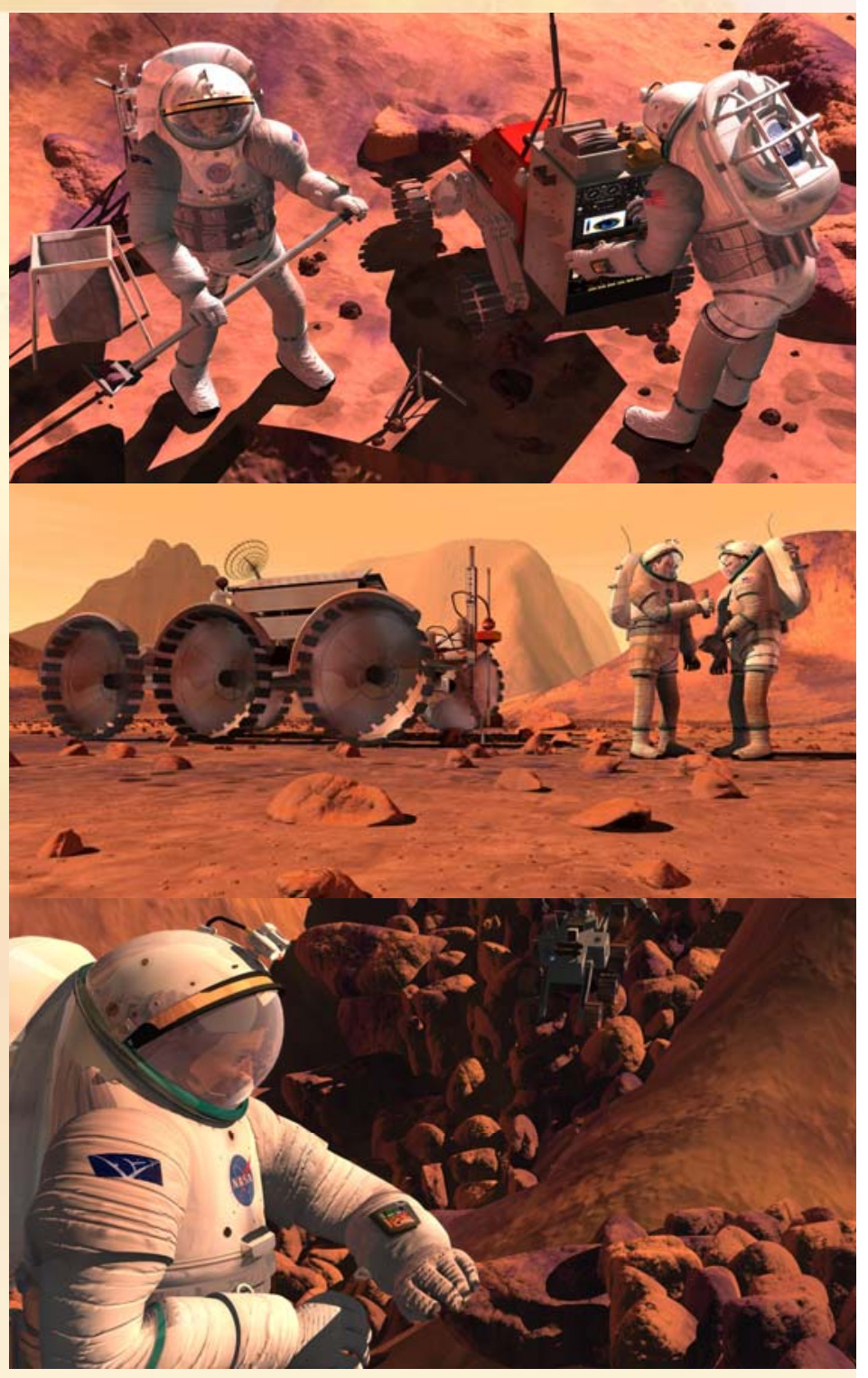




\section{Mars Design Reference Architecture 5.0 Example Long-Range Exploration Scenario}

- Landing site in a "safe" but relatively uninteresting location

- Geologic diversity obtained via exploration range

- Example case studies developed to understand exploration capability needs

- RED line indicates a set of example science traverses

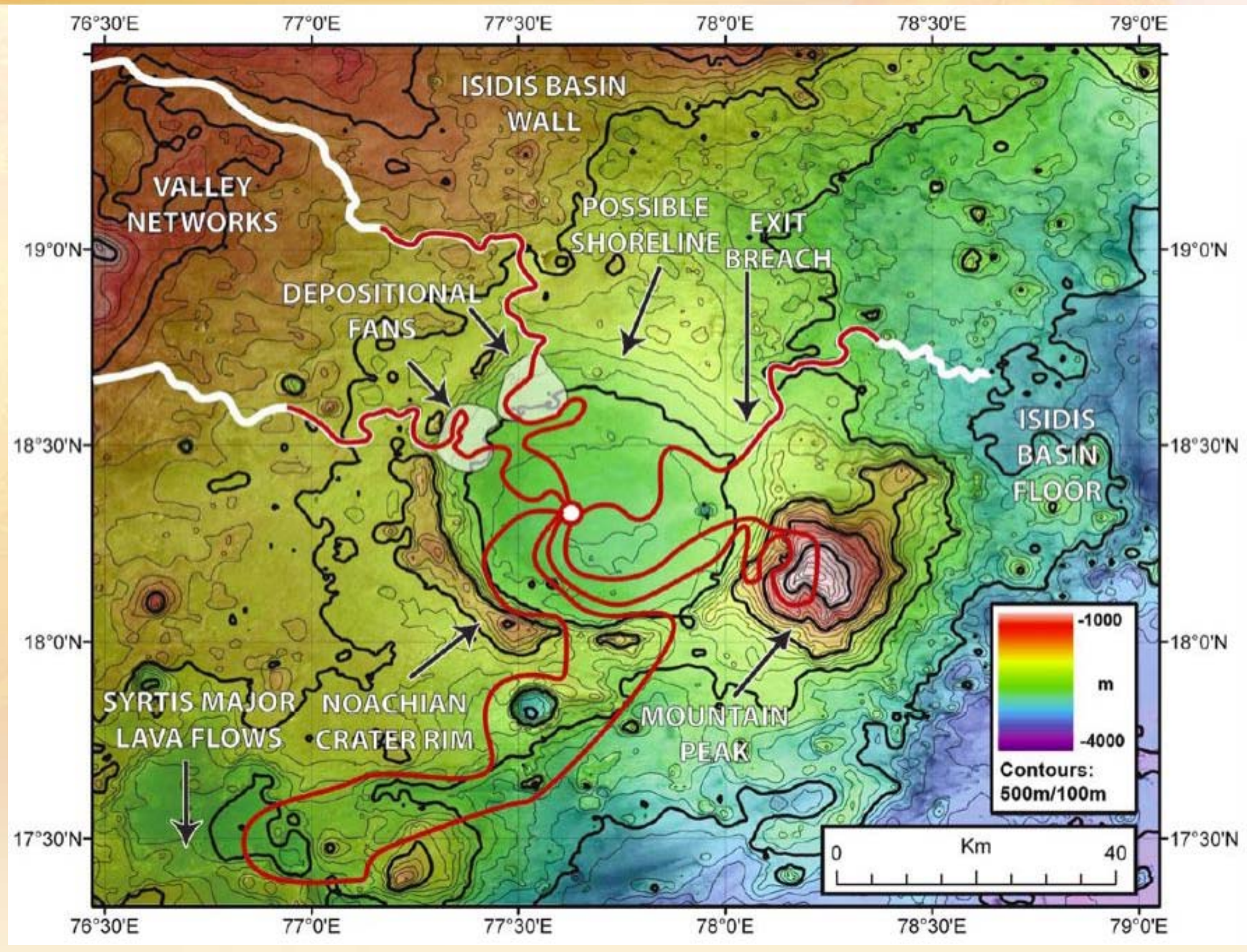




\section{Mars Design Reference Architecture 5.0 Planetary Protection}

- NASA Planetary Protection Policy is consistent with the COSPAR policy and is documented in NASA Policy Directive NPD 8020.7

- Specific requirements for human missions have not yet been issued.

- "Special regions" are areas that can be identified as being especially vulnerable to biological contamination and requiring special protections

"A region within which terrestrial organisms are likely to propagate, OR A region that is interpreted to have a high potential for the existence of extant Martian life forms. "COSPAR 2002

- "Zones of Minimum Biological Risk" (ZMBRs) are regions that have been demonstrated to be safe for humans.

- Astronauts will only be allowed in areas that have been demonstrated to be safe.

- Exploration plans and systems must be designed to maximize exploration efficiency while maintaining effective planetary protection controls

- Sterilization of hardware

- Minimized contamination release from human systems into the environment

- Human/robotic partnerships

- Human health monitoring

- Safeguarding the Earth, and by extension astronauts, from harmful backward contamination must always be the highest planetary protection priority

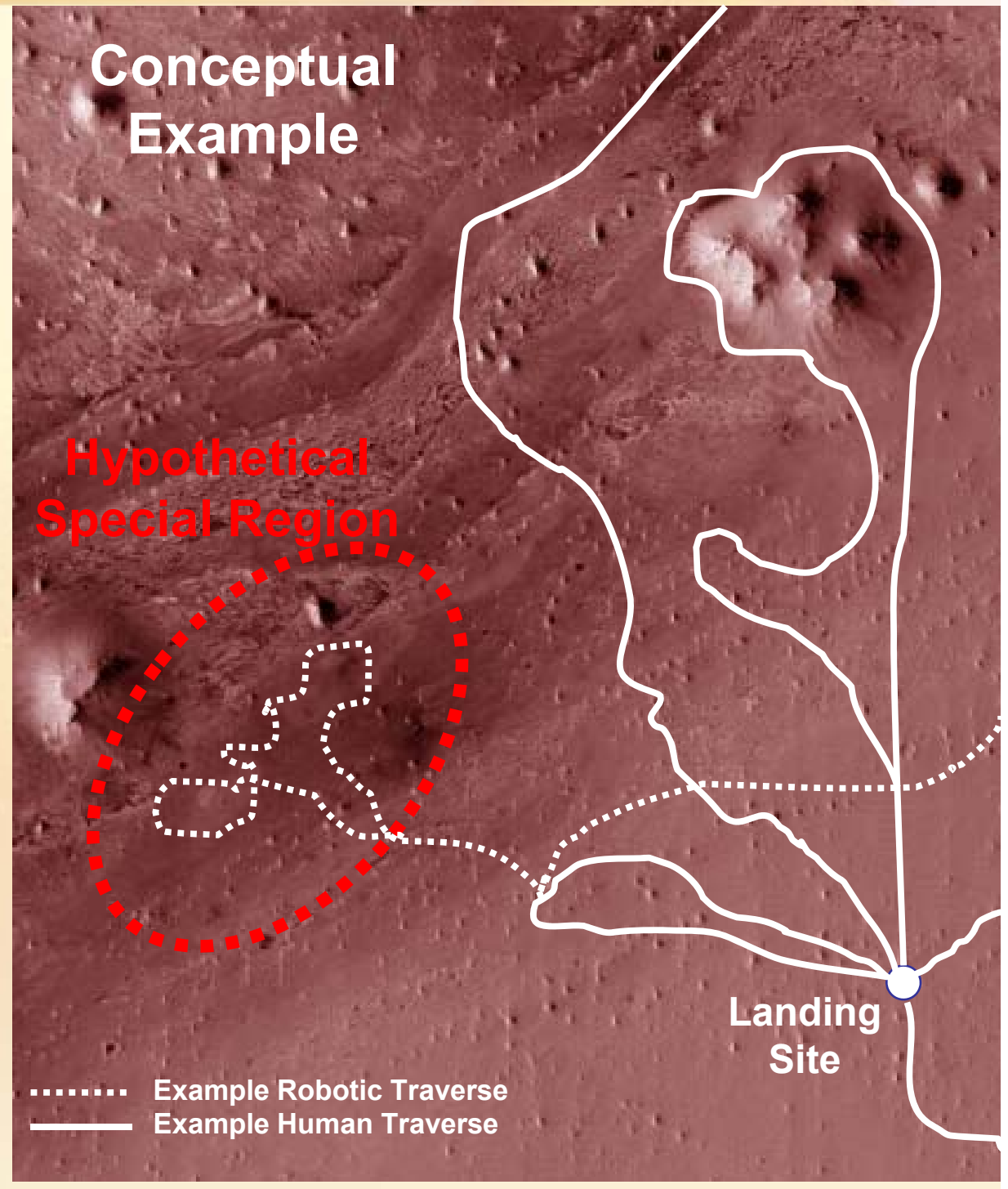




\section{Mars Design Reference Architecture 5.0 Evolutionary Testing Strategy}

\section{Earth/ISS}

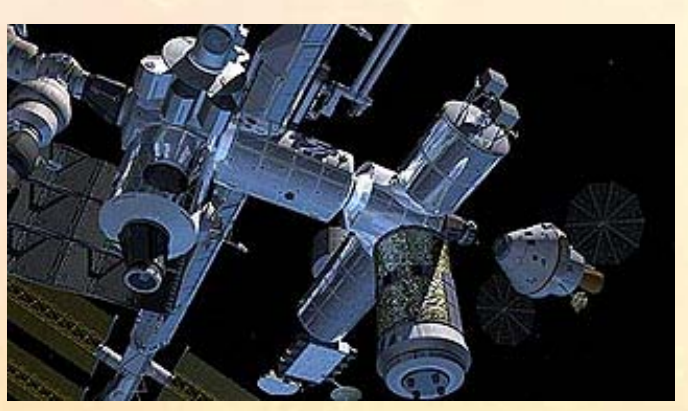

- Critical long-duration performance data of both hardware and operational concepts

- Validation of gravity-sensitive phenomena (crew physiology, gas/liquid separation, large scale structure deployments, etc.)

- Venue for long-duration system testing including crew interaction with hardware, software, and operational procedures such as lowest level component repair

- Simulation of operational concepts, such as vehicle deployment and assembly, prior to commitment to final vehicle design and operational mission concept

- Long-term exposure of systems to the deep-space environment, including radiation and zero-g can be conducted on missions in nearEarth space

January 15, 2009

\section{Knowledge / Experience / Confidence}

Moon

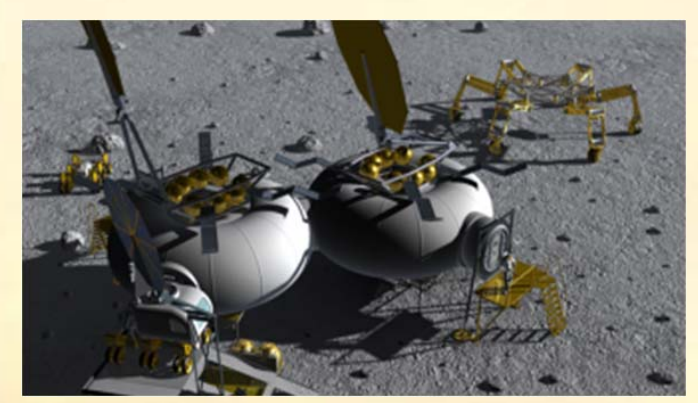

- Demonstration and use of Mars prototype systems (habitation, power, ISRU, mobility, etc.) to enhance lunar capabilities while improving confidence in future Mars systems

- Commonality and lowest level maintenance and repair concepts and technologies

- Surface exploration scenarios and techniques

- Long-term exposure of systems to the deep-space environment including radiation and dust

- Long-term "dry run" rehearsals and "what if" scenarios for future human Mars missions

\section{Mars via Robotics}

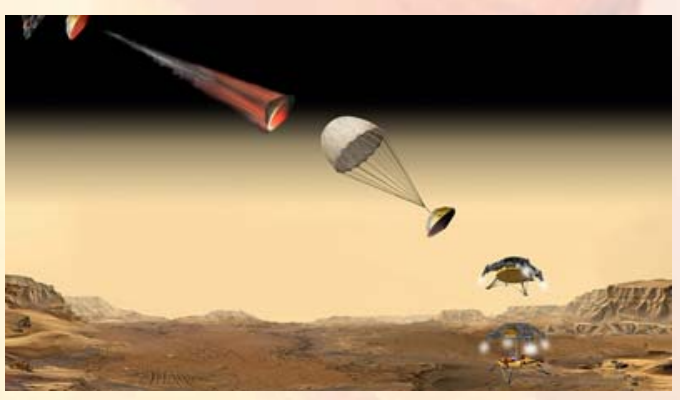

- Gathering environmental data of Mars (dust composition, thermal, radiation, terrain, hazards, etc.)

- Demonstration of integrated aeroassist technologies system performance

- Advanced technology demonstrations applicable to future human missions (e.g. IVHM, ISRU, power, thermal management, etc.)

- Dust mitigation techniques

- Large-scale robotic missions can demonstrate nuclear power components and systems operational characteristics, landing dynamics and physics (cratering), as well as serve to pre-deploy future human mission assets

- Large-scale unmanned cargo missions which land prior to the human mission can certify human landing vehicles 


\section{Mars Design Reference Architecture 5.0 Key Driving Requirements (KDR) \& Challenges}

\section{- Ground Ops}

- 7+ launches per mission

- 30 day launch centers (300 day launch campaign)

- Processing of nuclear systems

- Ares-V launch vehicle configuration

- Production and storage of cryogenics and helium

- Ares-V

- $10-\mathrm{m}$ dia $\times 30 \mathrm{~m}$ total length launch shroud

- Dual use shroud (EDL)

- $125+\mathrm{t}$ to LEO

- Launch to higher inclinations

- EDS evolution to long-duration (option)

- Cross-cutting

- Automated Rendezvous \& Docking (in Earth orbit)

- Cryogenic fluid management $\left(\mathrm{H}_{2}, \mathrm{O}_{2}, \mathrm{CH}_{4}\right)$

- Commonality \& lowest level maintenance \& repair

- Long-term system operation (300-1200 days)

- Low-Earth Orbit loiter for 300+ days

- Planetary protection

- Dust mitigation

- Mobility and Exploration

- $100+\mathrm{km}$ roving range

- $10+m$ depth access

- $\quad$ Light-weight, dexterous, maintainable EVA

- In-situ laboratory analysis capabilities
- Human Health \& Support

- Support humans in space for 900 days

- Radiation protection \& forecasting

- Zero-g countermeasures

- Closed-loop life support (air \& water)

- In-Space Transportation

- $\quad 50$ t roundtrip (LEO to Mars orbit return)

- $\quad 110-125 \mathrm{t}$ to Trans-Mars Injection

- Assembly via docking only

- ISRU compatible lander propulsion (oxygen)

- Integrated transportation flight experience

- Advanced Inter-planetary Propulsion

- Aeroassist

- 40-50 t payload to the surface

- Aerocapture + EDL for cargo

- Abort-to-Mars surface

- $12 \mathrm{~km} / \mathrm{s}$ Earth return speed

- Surface Related

- Auto-deployment and checkout of systems 30+ kWe continuous power

- Reliable back-up power system

- ISRU

- Extraction, storage and use of consumables from the martian atmosphere

- Production of $24 \mathrm{t}$ of oxygen for ascent

- Production of life support oxygen (2t) and water $(3.5 \mathrm{t})$ 


\section{Human Exploration of Mars Key Decisions and Tenets}

- Long surface stays with visits to multiple sites provides scientific diversity thus maximizing science return

- Mars systems pre-deployed to reduce mission mass and conduct system checkout prior to crew departure from Earth

- Enabling characteristics of human exploration of Mars:

- Entry, Descent, and Landing of large payloads (40 t) - Dual use Ares V shroud

- Robust Ares V launch campaign: 7+ launches on 30-day centers

- Nuclear Thermal Rocket (NTR) propulsion preferred transportation option (retain chemical/aerobrake as backup)

- ISRU : Production of ascent propellant (oxygen) and crew consumables from the atmosphere

- Nuclear surface power : Enables In-Situ Resource Utilization (ISRU) while providing continuous robust power

- Mobility at great distances (100's km) from the landing site enhances science return (diversity)

- A rich "Mars like" lunar Program which demonstrates key system behavior, operability, repair, and time on systems is necessary

- Operation and maintenance of systems for long durations (500-1200 days) with no logistics resupply 